\title{
A Novel approach to FePt assemblage and
}

\section{synthesis}

Rebecca O. Fuller ${ }^{\bullet l, 2}$, Nicole S. Hondow ${ }^{1}$, George A. Koutsantonis ${ }^{1}$, Martin Saunders ${ }^{3}$ and Robert L. Stamps ${ }^{2}$

${ }^{1}$ Chemistry, M313, School of Chemical and Biomedical Sciences, University of Western Australia, 35 Stirling Highway, Crawley, Western Australia 6009 Australia

${ }^{2}$ School of Physics, M013, University of Western Australia, 35 Stirling Highway, Crawley, Western Australia 6009 Australia

${ }^{3}$ Centre for Microscopy, Characterisation and Analysis, M010, University of Western Australia, 35 Stirling Highway, Crawley, Western Australia 6009 Australia

- Corresponding author. Email: becky@physics.uwa.edu.au Tel: +61-8-9-6488-4441 Fax:+61-8-6488-7247 
Qualitative EDS analysis has been used to confirm the presence of both Fe and Pt in the particles synthesized in the presence of MCM-41. An example spectrum from a sample mounted on a carbon coated copper grid is shown below in Figure 1. Peaks due to the presence of elemental $\mathrm{Si}, \mathrm{O}, \mathrm{Fe}, \mathrm{Pt}$ and $\mathrm{Cu}$ are seen.

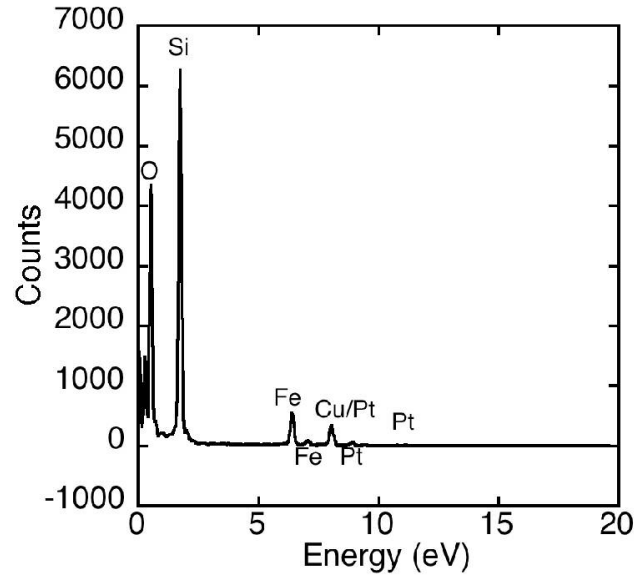

a

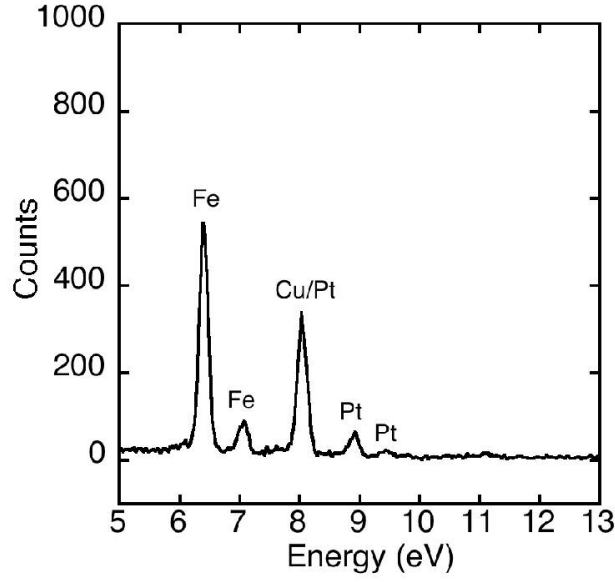

b

Figure 1: Full EDS spectrum (a) for FePt nanoparticles synthesized in-situ MCM-41. A close up of the peaks assigned to the nanoparticles is shown in (b) 